\title{
Data Rate Limit in Low and High SNR Regime for Nakagami-q Fading Wireless Channel
}

\author{
Md. Mazid-Ul-Haque ${ }^{1}$, Md. Sohidul Islam ${ }^{2}$ \\ Department of Computer Science \\ American International University-Bangladesh \\ Dhaka, Bangladesh
}

\begin{abstract}
Adequate data rate is always desired in wireless communication channels. Previously, few fading models were used to model wireless communication channels and to perform analysis on them. In this paper, analyses of data rate limit of single-input single-output (SISO) wireless communication system over Nakagami-q fading channels are presented. The calculation of capacity has been carried out using small and large limit argument approximations. The analytical solution for channel capacity is presented using small and large limit argument approximations. Where small and large limit argument approximations correspond low and high signal-to-noise ratio (SNR) regime. Behavior of channel capacity with respect to SNR and fading parameter respectively has been investigated deeply. The comparison of the channel capacity behavior for both low SNR and high SNR regime and have also been done and analyzed. It has found that the channel capacity increased with increasing SNR in low SNR regime. The channel capacity also behave in the same manner in high SNR regime as well.
\end{abstract}

Keywords-Wireless communication; Nakagami-q fading; SISO channel capacity; low SNR regime; high SNR regime

\section{INTRODUCTION}

Having a good data transfer rate in wireless communication system is always expected for users. Downloading and uploading information faster is becoming more and more demandable with the advancement of technology. In order to do so, the capacity of the wireless communication system needs to be increased. Shannon [1], first introduced the concept of information theoretic capacity. Essential outlines of communication over wireless channel was characterized in [1] by Shannon. Since then many wide research has been done on information capacity for different distributions. Systems evolved from single-input single-output (SISO) to multipleinput multiple-output (MIMO) over the time period. In [2], authors discussed about SISO and MIMO channel capacities. They compared SISO capacities among different distributions which includes Uniform distribution, Gaussian distribution, Chi-square distribution [2]. Authors evaluated the performance of SISO, SIMO and MIMO antenna systems in [3]. In [4], authors presented results based on their findings on the capacity of discrete-time Rayleigh-fading SISO and MIMO channels.

Nakagami-m, Rician and Rayleigh distributions are popular distribution models for research on SISO, SIMO, MIMO channels. Besides these distribution there is Nakagami-q distribution also known as Hoyt distribution which is a type of fading distribution that under indisputable conditions serves a decent model. In [5], authors state that this distribution permits the modeling of the environment of propagation without a presiding component over the scattered waves which is also known as a situation of Non-line of Sight. Over generalized fading channel, it has been found that, Nakagami-q distribution has application in the error execution assessment of digital correspondence system [6]. Likewise, this fading channel is usually observed in satellite link subject to powerful ionospheric glimmer and intensely shadowed condition [7]. In inter-satellite communications, instantaneous signal to noise ratio follows the log-square-Hoyt distribution [8]. For performance analysis and other studies which are related to mobile radio communications, the Nakagami-q model is being used more frequently.

In this research work, study of the data rate limit of SISO wireless communication system over this Nakagami-q fading channels is done. For this, the calculation of the capacity for low and high SNR regimes based on Nakagami-q distributions including the comparison of them have been done in order to analyze how consistent and good the data rate limits are with respect to instantaneous SNR and fading parameter.

The rest of the paper contains the followings. Nakagamiq distribution in Section 2. System model for this study in Section 3. In Section 4, the capacity calculations for low and high SNR regimes for Nakagami-q fading wireless channels. Section 5 contains the results and analysis. At last, the concluding remarks of this work are presented in Section 6.

\section{NAKAGAMI-Q DisTRIBUTION}

Nakagami-q distribution or Hoyt distribution is usually used to depict the transient signal variation of certain wireless communications systems subject to fading [9]. This distribution can be defined as the distribution of modulus of complex Gaussian random variables with zero mean and unequal variances [10]. The probably distribution function simplified as PDF, of this Nakagami-q distribution is given in [11] by,

$$
p_{\gamma}(\gamma)=\frac{\left(1+q^{2}\right) \gamma}{2 q \bar{\gamma}} e^{\frac{-\left(1+q^{2}\right)^{2} \gamma}{4 q^{2} \bar{\gamma}}} I_{0}\left(\frac{\left(1-q^{4}\right) \gamma}{4 q^{2} \bar{\gamma}}\right), \quad \gamma \geq 0
$$

Here, $I_{0}($.) represents the modified Bessel function of the first kind of zeroth-order. Nakagami-q fading parameter is represented as $\mathrm{q}$. The range of the fading parameter is from 0 to 1 . And $\gamma$ is the instantaneous SNR and $\bar{\gamma}$ is the average SNR. When $\mathrm{q}=0$, it is one sided Gaussian fading and for $\mathrm{q}=1$, it becomes Rayleigh fading. 


\section{SySTEM Model}

In single-input single-output (SISO) wireless communication system, both the transmitter and the receiver are equipped with single transmitting and single receiving antenna respectively.

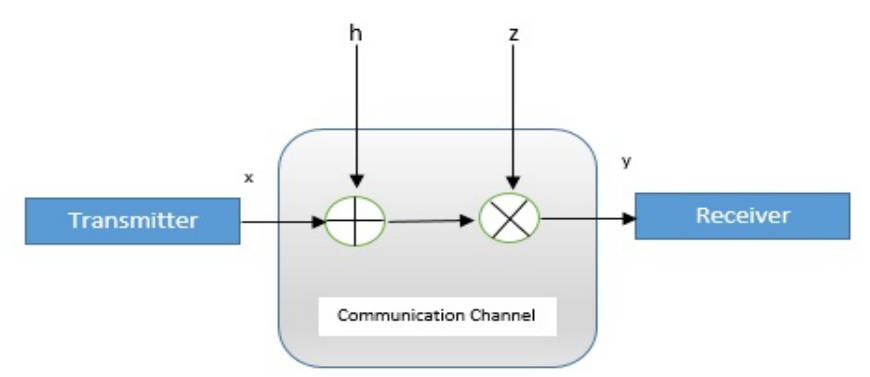

Fig. 1. System Model for Nakagami-q Fading SISO Wireless Channel.

The SISO system model for this work is shown in Fig. 1. For this study, let us consider $\mathrm{Pt}$ as transmitted signal power and let us also consider at the receiver end the received signal being corrupted by AWGN (additive white Gaussian Noise) also the transmission is carried over Nakagami-q fading channel. As described in [12] the received signal vector can be expressed as,

$$
r=h x+z
$$

Here, $\mathrm{h}$ indicating a vector that defines the channel gains from transmitter to the receiver antenna, $x$ indicates the transmitted signal and $\mathrm{z}$ is a vector of zero mean complex AWGN. For SISO wireless communication systems, [13] states that, Shannon channel capacity is the highest reciprocated information between the transmitted signal and the received one and the capacity can be illustrated as,

$$
C=W \log _{2}(1+\rho)
$$

Here, $\rho=\frac{P_{t}}{\sigma^{2}}$ is the transmitted signal to noise ratio while $\mathrm{W}$ indicates the transmission bandwidth. As said in [10] and [14], the channel is power restricted in the perception that, $p_{t}=E\left\{|x|^{2}\right\}$ where the expectation operator is denoted by $\mathrm{E}\{$.$\} that is evaluated over the probability distribution function$ (pdf) of channel gain matrix, h.

For low SNR regime, using small argument limit approximation as described in [15], the zeroth-order modified Bessel function of the first kind can be approximated as $I_{0} \approx 1$.

Using the above approximation the distribution in (1) becomes,

$$
p_{\gamma}(\gamma)=\frac{\left(1+q^{2}\right) \gamma}{2 q \bar{\gamma}} e^{\frac{-\left(1+q^{2}\right)^{2} \gamma}{4 q^{2} \bar{\gamma}}}, \gamma \geq 0
$$

Eq. (4) is the Nakagami-q distribution under small limit argument approximation for low SNR regime. Eq. (4) becomes Rayleigh fading distribution if the fading parameter is set $\mathrm{q}=1$.

For high SNR regime, the derivation of the expression for the Nakagami-q distribution using large argument approximation is done. According to [16], the zeroth order modified Bessel function can be written as,

$$
I_{0}(r t) \approx \frac{e^{r t}}{\sqrt{2 \pi r t}}, r t>>1
$$

So, using this large argument approximation for Eq. (1), the zeroth order modified Bessel function of the Nakagami-q distribution becomes,

$$
I_{0}\left(\frac{\left(1-q^{4}\right) \gamma}{4 q^{2} \bar{\gamma}}\right) \approx \frac{e^{\frac{\left(1-q^{4}\right) \gamma}{4 q^{2} \bar{\gamma}}}}{\sqrt{2 \pi \frac{\left(1-q^{4}\right) \gamma}{4 q^{2} \bar{\gamma}}}}
$$

Nakagami-q distribution given in Eq. (1) using this above approximation becomes,

$$
p_{\gamma}(\gamma)=\frac{\left(1+q^{2}\right) \gamma}{2 q \bar{\gamma}} e^{\frac{-\left(1+q^{2}\right)^{2} \gamma}{4 q^{2} \bar{\gamma}}} \frac{e^{\frac{\left(1-q^{4}\right) \gamma}{4 q^{2} \bar{\gamma}}}}{\sqrt{2 \pi \frac{\left(1-q^{4}\right) \gamma}{4 q^{2} \bar{\gamma}}}}, \gamma \geq 0
$$

To simplify Eq. (4) and (7) let us consider

$$
a=\frac{1+q^{2}}{2 q \bar{\gamma}}, b=\frac{\left(1+q^{2}\right)^{2}}{4 q^{2} \bar{\gamma}} \text { and } n=\frac{1-q^{4}}{4 q^{2} \bar{\gamma}}
$$

Using the (8) simplifications, Eq. (4) becomes,

$$
p_{\gamma}(\gamma)=a \gamma e^{-b \gamma}, \quad \gamma \geq 0
$$

And using the (8) simplifications Eq. (7) becomes,

$$
p_{\gamma}(\gamma)=a \gamma e^{-b \gamma} \frac{e^{n \gamma}}{\sqrt{2 \pi n \gamma}}, \quad \gamma \geq 0
$$

Eq. (10) represents the Nakagami-q distribution for high SNR regime under large argument approximation.

Eq. (9) and (10) are considered as Nakagami-q distribution for low SNR and high SNR regimes, respectively. 


\section{CAPACITy CAlculation}

In this section the calculation of the capacity for Nakagami$\mathrm{q}$ fading SISO channels is done for low SNR regime and high SNR regime. The capacity of this system is obtained by using Eq. (3),

$$
C=\int_{0}^{\infty} p_{\gamma}(\gamma) \log _{2}(1+\rho \gamma) d \gamma
$$

For low SNR regime substituting Eq. (9) into Eq. (11), the capacity becomes,

$$
C=\int_{0}^{\infty} a \gamma e^{-b \gamma} * \log _{2}(1+\rho \gamma) d \gamma
$$

and substituting Eq. (10) into Eq. (11), the capacity for high SNR regime becomes,

$$
C=\int_{0}^{\infty} a \gamma e^{-b \gamma} \frac{e^{n \gamma}}{\sqrt{2 \pi n \gamma}} * \log _{2}(1+\rho \gamma) d \gamma
$$

Mathematica [17], an advanced technical computing system is to explicate and validate Eq. (12) and (13) in order to analyze the data rate limit of this in high and low SNR regimes. in,

Using Eq. (12) the capacity of the low SNR regime results

$$
C_{L S R}=\frac{a}{\rho^{2} \log [2]}\left(G_{2,3}^{3,1}\left(\left.\frac{b}{\rho}\right|_{-2,-2,0} ^{-2,-1}\right)\right)
$$

if $\operatorname{Re}[\mathrm{b}]>0$ and $\operatorname{Re}[\rho]>0$, where $G_{p, q}^{m, n}\left(\left.x\right|_{b_{q}} ^{a_{p}}\right)$ is the Meijer G-function and $C_{L S R}$ is the capacity of low SNR regime.

And using Eq. (13) the capacity of the high SNR regime results in,

$$
\begin{gathered}
C_{H S R}=\frac{a e^{\frac{-n}{\rho}}}{\left(2 \sqrt{2}(b-n)^{3 / 2} \sqrt{n} \rho \log [2]\right)} \\
\left(\left(-2 e^{n / \rho} \rho+e^{n / \rho} 0.577216 \rho+2 e^{b / \rho} \sqrt{b-n} \sqrt{\rho} \sqrt{\pi}\right.\right. \\
-e^{n / \rho} \rho \pi\left(\operatorname{Erfi}\left[\frac{\sqrt{b-n}}{\sqrt{\rho}}\right]\right) \\
+2 e^{n / \rho}(-b+n)\left(\left({ }_{2} F_{2}\right)\left(1,1 ; \frac{1}{2}, 2 ; \frac{b-n}{\rho}\right)\right)+e^{n / \rho} \rho \log [4] \\
\left.\left.+e^{n / \rho} \rho \log [b-n]-e^{n / \rho} \rho \log [\rho]\right)\right)
\end{gathered}
$$

if $\operatorname{Re}[b-n]>0$, where 0.577216 is the "EulerGamma" which is a mathematical constant and Erfi (x) is the imaginary error function and ${ }_{p} F_{q}(\mathrm{a} ; \mathrm{b} ; \mathrm{z})$ is the hypergeometric function and the generalized form is given by
HypergeometricPFQ $\left[\left\{a_{1}, \ldots, a_{p}\right\},\left\{b_{1}, \ldots, b_{q}\right\}, z\right]$ and $C_{H S R}$ is the capacity of high SNR regime..

In the next section the plotting of Eq. (14) and (15) are done for some certain values of fading parameter, average SNR, instantaneous SNR and the analyses of the data rate limit are presented also.

\section{CAPACITY ANALYSIS}

This analysis section is divided into two subsections. Behavior of capacity with respect to the instantaneous SNR and the fading parameter respectively is presented here and comparison of the capacity behavior for low SNR regime and high SNR regime is done in this section.

\section{A. Data Rate Limit with Respect to Instantaneous SNR}

First, plotting of the capacity vs instantaneous SNR for low SNR regime is done then the next is potted which is capacity vs instantaneous SNR for high SNR regime and at last comparison of both the low and the high SNR regime scenarios is done. Following Fig. 2 presents the capacity behavior with respect to instantaneous SNR for low SNR regime.

In Fig. 2 the correctness of Eq. (14) is verified which is derived from Eq. (12) by plotting Eq. (14) and Eq. (12) together. The same values are used for Eq. (12) as in Eq. (14).

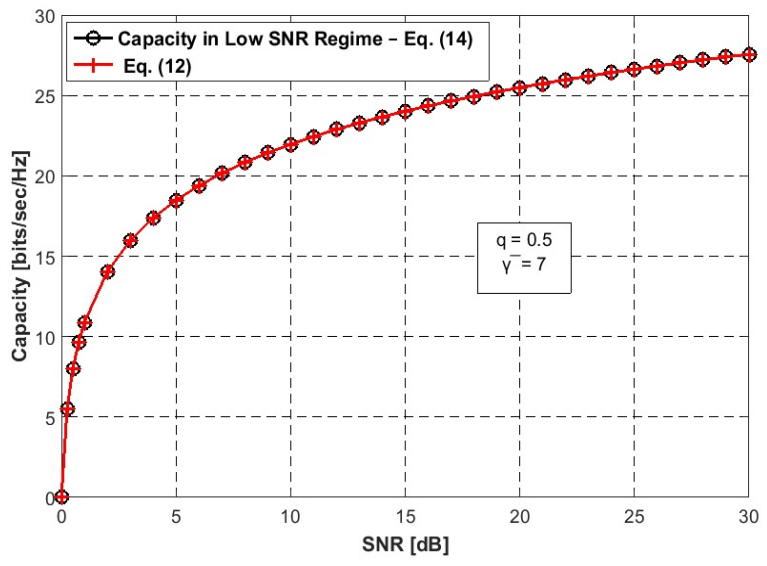

Fig. 2. Capacity [bits/sec/Hz] for low SNR regime with respect to instantaneous SNR $[\mathrm{dB}]$.

It can be seen in Fig. 2 that Eq. (14) is the accurate derivation of Eq. (12) as both the equations generate the exact same curve for the exact same values.

From Fig. 2 it is found that capacity of this Nakagami-q fading SISO system is increasing along with the increasing instantaneous signal to noise ratio.

Fig. 2 represents the capacity of this system with fading parameter $\mathrm{q}=0.5$, and average signal to noise ratio $=7$. In this scenario capacity at $1 \mathrm{~dB}$ is $10.8631 \mathrm{bits} / \mathrm{sec} / \mathrm{Hz}$ and as the capacity increases with the increasing of instantaneous SNR, the capacity at $30 \mathrm{~dB}$ is found $27.5641 \mathrm{bits} / \mathrm{sec} / \mathrm{Hz}$.

Fig. 3 presents the capacity behavior with respect to instantaneous SNR for high SNR regime. 
As done for the previous low SNR scenario, the verification of the correctness of Eq. (15) is also done which is derived from Eq. (13) by plotting Eq. (15) and Eq. (13) together using same values for Eq. (13) as in Eq. (15) for high SNR scenario in Fig. 3.

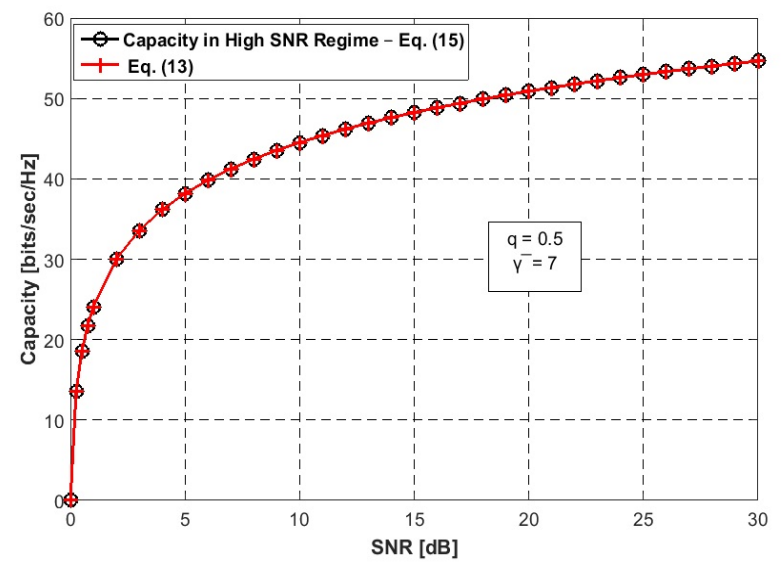

Fig. 3. Capacity [bits/sec/Hz] for high SNR regime with respect to instantaneous SNR [dB].

The justification of Eq. (15) as the accurate derivation of Eq. (13) can be seen in Fig. 3 as both the equations produce the exact same curve for the exact same values.

From Fig. 3 it is also found that in high SNR regime capacity of this Nakagami-q fading SISO system is increasing along with the increasing instantaneous signal to noise ratio.

Fig. 3 represents the capacity of this system with fading parameter $\mathrm{q}=0.5$, and average signal to noise ratio $=7$. In this scenario capacity at $1 \mathrm{~dB}$ is $24.0783 \mathrm{bits} / \mathrm{sec} / \mathrm{Hz}$ and the capacity at $30 \mathrm{~dB}$ is found $54.66 \mathrm{bits} / \mathrm{sec} / \mathrm{Hz}$.

Now the comparison of both the high and low SNR regime capacity is done with the same value of fading parameter $\mathrm{q}=$ 0.5 , and average signal to noise ratio $=7$. Fig. 4 presents the capacity of this system in both high and low SNR regimes with respect to instantaneous SNR.

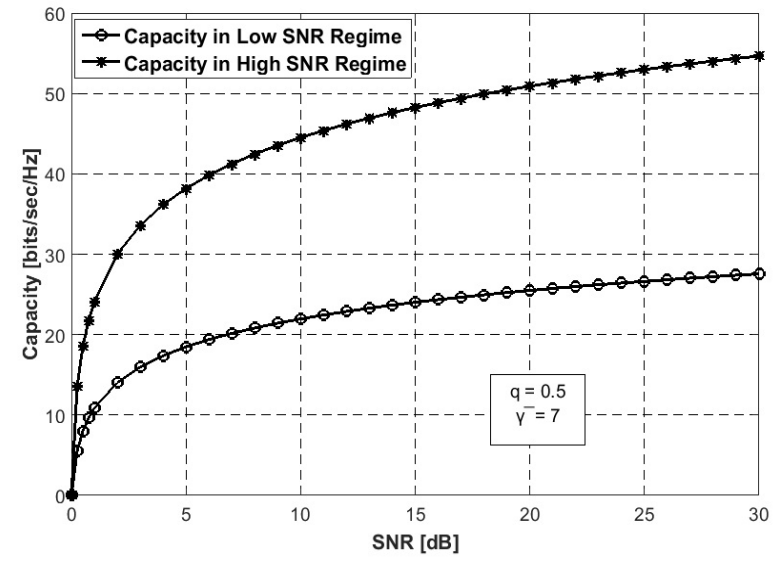

Fig. 4. Capacity [bits/sec/Hz] for both low and high SNR regimes with respect to instantaneous SNR $[\mathrm{dB}]$.
It is found from Fig. 2 and Fig. 3 that for both low SNR and high SNR regimes capacity of this system is increasing along with the increasing instantaneous signal to noise ratio. From Fig. 4 it can be stated that the capacity of this system has a higher increasing rate in high SNR regime than that of the low SNR regime.

In this scenario capacity at $1 \mathrm{~dB}$ is $10.8631 \mathrm{bits} / \mathrm{sec} / \mathrm{Hz}$ for low SNR regime and $24.0783 \mathrm{bits} / \mathrm{sec} / \mathrm{Hz}$ for high SNR regime and the capacity at $30 \mathrm{~dB}$ is $27.5641 \mathrm{bits} / \mathrm{sec} / \mathrm{Hz}$ for low SNR regime and $54.66 \mathrm{bits} / \mathrm{sec} / \mathrm{Hz}$ for high SNR regime. There is about $90.3197 \%$ increase of capacity for the range of 0 to 30 $\mathrm{dB}$ SNR in high SNR regime than the low SNR regime.

So, it can be said from the above analyses is that this system has higher data rate in high SNR regime than the data rate of the low SNR regime.

\section{B. Data Rate Limit with Respect to Fading Parameter}

In this subsection, plotting of the capacity vs fading parameter for low SNR regime is done first then moving to the next regime which high SNR regime and at last, like the previous subsection a comparison is presented of both the low and the high SNR regime scenarios.

Fig. 5 presents the capacity behavior with respect to fading parameter for low SNR regime.

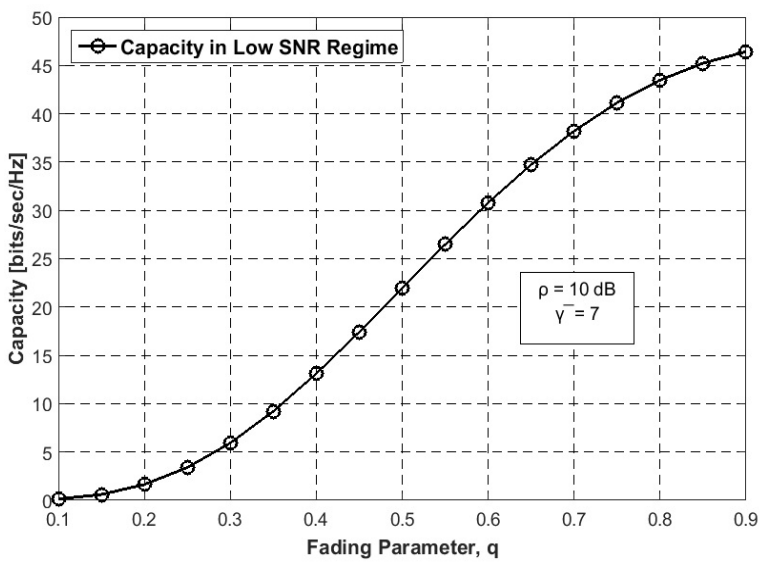

Fig. 5. Capacity [bits/sec/Hz] for low SNR regime with respect to fading parameter, q.

From above Fig. 5 it is found that capacity is increasing along with the increasing fading parameter, $q$. Up to 0.2 value of fading parameter there is a small increase in capacity and for the value of fading parameter after 0.2 up to 0.9 the increase is logistic.

Fig. 5 represents the capacity of this Nakagami-q fading SISO system with instantaneous SNR $=10 \mathrm{~dB}$, and average signal to noise ratio $=7$. In this case capacity at $q=0.1$ is $0.133391 \mathrm{bits} / \mathrm{sec} / \mathrm{Hz}$ and as the capacity increases with the increasing of fading parameter, the capacity at $\mathrm{q}=0.9$ is found $46.4261 \mathrm{bits} / \mathrm{sec} / \mathrm{Hz}$.

Fig. 6 represents the capacity behavior with respect to fading parameter for high SNR regime. 


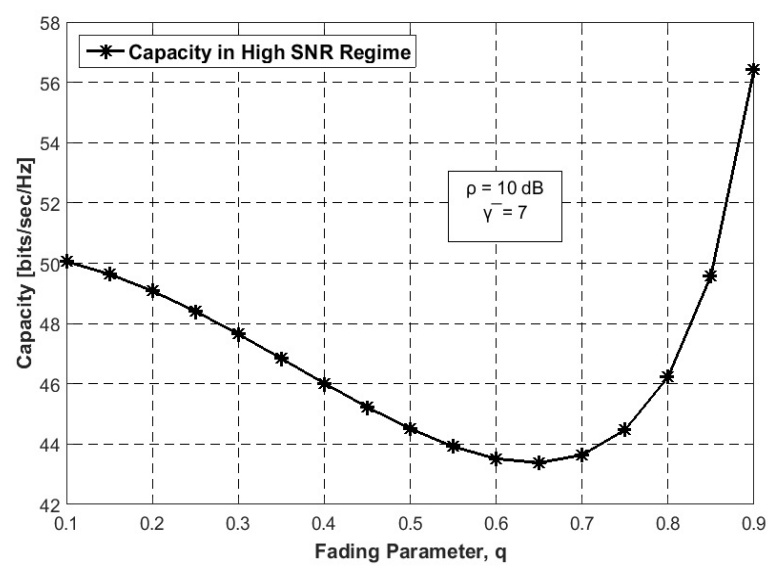

Fig. 6. Capacity [bits/sec/Hz] for high SNR regime with respect to fading parameter, q.

From Fig. 6 it is found that in high SNR regime capacity of this Nakagami-q fading SISO system has a different characteristics than that of the low SNR regime. The capacity in this high SNR regime is first decreasing along with the increasing fading parameter, $\mathrm{q}$, starting from $\mathrm{q}=0.1$ up to $\mathrm{q}=0.65$. And from $\mathrm{q}=0.66$ up to $\mathrm{q}=0.9$ there is exponential increase of capacity.

Fig. 6 presents the capacity of this system with instantaneous $\mathrm{SNR}=10 \mathrm{~dB}$, and average signal to noise ratio $=7$. In this scenario capacity at $\mathrm{q}=0.1$ is $50.0579 \mathrm{bits} / \mathrm{sec} / \mathrm{Hz}$, capacity at $\mathrm{q}=0.65$ is $43.3829 \mathrm{bits} / \mathrm{sec} / \mathrm{Hz}$ and the capacity at $\mathrm{q}=0.9$ is found $56.4169 \mathrm{bits} / \mathrm{sec} / \mathrm{Hz}$.

And now, comparison of both the high and low SNR regime capacity is done with the same value of instantaneous SNR = $10 \mathrm{~dB}$, and average signal to noise ratio $=7$. Fig. 7 presents the capacity of this system in both high and low SNR regimes with respect to fading parameter, q.

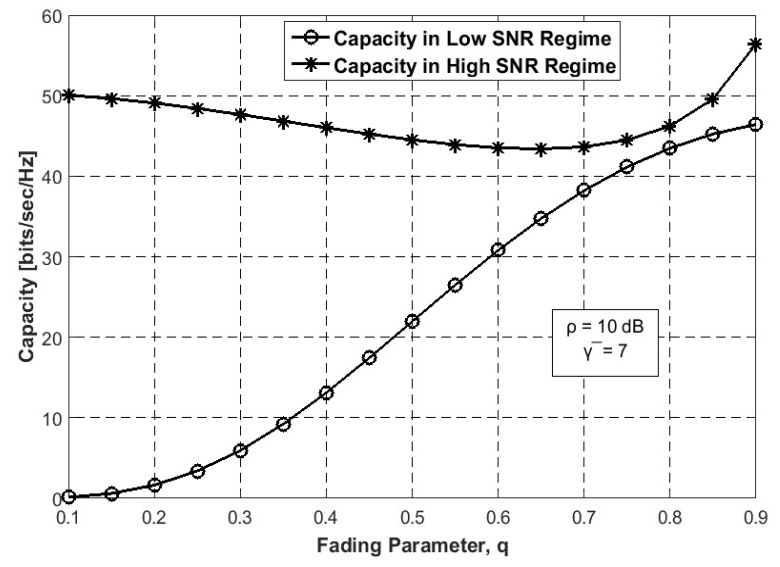

Fig. 7. Capacity [bits/sec/Hz] for both low and high SNR regimes with respect to fading parameter, $\mathrm{q}$.

In Fig. 7, it is found from the comparison that the data rate in this case is higher in high SNR regime as the capacity with respect to the fading parameter has a higher value for the high SNR regime than the capacity of this system in low SNR regime. But it is also found that the capacity growth is consistent in low SNR regime than in high SNR regime as the capacity in high SNR regime is decreasing first up to a certain value of fading parameter and then it is increasing rapidly but in low SNR regime capacity increases logistically with the increasing of fading parameter.

There is no point of intersection found for capacity in Fig. 6. The separating value of capacity for this case is 43.4795 to $46.2262 \mathrm{bits} / \mathrm{sec} / \mathrm{Hz}$.

\section{CONCLUSiON}

In this paper, study of the data rate limit in both high and low SNR regime for Nakagami-q fading SISO wireless channel is discussed. For this the novel analytical expressions for the capacity of both the high and the low SNR regimes for this system using small limit and large limit argument approximation respectively are derived. This study presented in depth capacity analyses for both low and high SNR regimes. In this study, it is found that data rate of high SNR regime is better than low SNR regime as the capacity with respect to the instantaneous SNR for the system has a higher value in high SNR regime. It is also found that the capacity with respect to the fading parameter has a higher value in the high SNR regime but there is a noticeable fluctuation in this case as there is a notable decrease up to some point and after that the growth is exponential and in low SNR regime the capacity growth is logistic.

\section{REFERENCES}

[1] C. Shannon, "Communication theory of secrecy systems," Bell System Technical Journal, vol. 29, pp. 656-715, 1949.

[2] A. a. V. R. Khan, "A Tutorial on SISO and MIMO Channel Capacities," in Khan, A. and Vesilo, R., A Tutorial on SISO anDepartment of Electronics, Macquarie University NSW, Sydney Australia.

[3] C. S. a. T. Z. L. Reichardt, "Performance evaluation of SISO, SIMO and MIMO antenna systems for car-to-car communications in urban environments," in 9th International Conference on Intelligent Transport Systems Telecommunications, (ITST), IEEE, Lille, 2009.

[4] L. W. B. H. a. A. L. V. Sethuraman, "Low-SNR Capacity of Noncoherent Fading Channels," IEEE Transactions on Information Theory, vol. 55, no. 4, pp. 1555-1574, 2009.

[5] R. S. D. S. A. Fasolo, "Fading channel simulator for Hoyt distribution," in Instituto Nacional de Telecomunicacoes, Brasil.

[6] O. L. a. J. M. C. G. Fraidenraich, "On the MIMO channel capacity for the dual and asymptotic cases over Hoyt channels," IEEE Communications Letters, vol. 11, no. 1, pp. 31-33, 2007.

[7] C.-X. W. a. M. P. N. Youssef, "A study on the second order statistics of Nakagami-Hoyt mobile fading channels," IEEE Transactions on Vehicular Technology, vol. 55, no. 4, pp. 1259-1265, 2005.

[8] X. Liu, "Optimization of Satellite Optical Transmission Subject to Log-Square-Hoyt Fading," in 2011 IEEE International Conference on Communications (ICC), Kyoto, 2011.

[9] J. F. Paris, "Nakagami-q (Hoyt) distribution function with applications," Electronics Letters, vol. 45, no. 4, pp. 210-211, 2009.

[10] M. R. I. Md. Sohidul Islam, "Ergodic Capacity of a SIMO System Over Nakagami-q Fading Channel," DUET Journal, vol. 2, no. 1, 2014.

[11] M. S. A. M. K. Simon, "Digital communication over fading channels," New York, 2000.

[12] A. M. M. a. M. M. Matalgah, "Capacity of SIMO systems over nonidentically independent Nakagami-m channels," in 2007 IEEE Sarnoff Symposium, Princeton, NJ, 2007. 
[13] J. G. Proakis, Digital Communication Systems, 4th edition, McGrawHill, 2001.

[14] M. S. I. and M. R. Islam, "Secrecy Mutual Information of the Independent and Identically Distributed Nakagami-q Fading SIMO Channel," IETE Journal of Research, vol. 65, no. 3, pp. 387-396, 2018.

[15] M. a. I. M. Islam, "Positive secrecy mutual information over nonidentically independently distributed Nakagami-q fading wireless chan- nel," in International Conference on Engineering, Research, Innovation and Education , 2013.

[16] Kölbig, K.S., A definite integral with modified Bessel functions (No. CERN-64-51). CERN, 1964.

[17] Wolfram Research, Inc., Mathematica, Version 12.0, Champaign, IL (2019). 